\title{
High Resolution Crystal Structures of the Cerebratulus lacteus Mini-Hb in the Unligated and Carbomonoxy States
}

Francesca Germani ${ }^{1}$, Alessandra Pesce ${ }^{2}$, Andrea Venturini ${ }^{2}$, Luc Moens ${ }^{1}$, Martino Bolognesi ${ }^{3}$, Sylvia Dewilde ${ }^{1, *}$ and Marco Nardini ${ }^{3, *}$

1 Department of Biomedical Sciences, University of Antwerp, Universiteitsplein 1, B-2610 Antwerp, Belgium; E-Mails: francesca.germani@ua.ac.be (F.G.); luc.moens@ua.ac.be (L.M.)

2 Department of Physics, University of Genova, Via Dodecaneso 33, I-16146 Genova, Italy; E-Mails: pesce@fisica.unige.it (A.P.); S2950466@studenti.unige.it (A.V.)

3 Dipartimento di BioScienze, Università degli Studi di Milano, Via Celoria 26, I-20133 Milano, Italy; E-Mail: martino.bolognesi@unimi.it

* Authors to whom correspondence should be addressed; E-Mails: sylvia.dewilde@ua.ac.be (S.D.); marco.nardini@unimi.it (M.N.); Tel.: +32-03-265-2323 (S.D.); +39-02-503-14898 (M.N.); Fax: +32-03-265-2339 (S.D.); +39-02-503-14895 (M.N.).

Received: 31 May 2012; in revised form: 14 June 2012 / Accepted: 15 June 2012 /

Published: 28 June 2012

Abstract: The nerve tissue mini-hemoglobin from Cerebratulus lacteus (CerHb) displays an essential globin fold hosting a protein matrix tunnel held to allow traffic of small ligands to and from the heme. CerHb heme pocket hosts the distal TyrB10/GlnE7 pair, normally linked to low rates of $\mathrm{O}_{2}$ dissociation and ultra-high $\mathrm{O}_{2}$ affinity. However, CerHb affinity for $\mathrm{O}_{2}$ is similar to that of mammalian myoglobins, due to a dynamic equilibrium between high and low affinity states driven by the ability of ThrE11 to orient the TyrB10 OH group relative to the heme ligand. We present here the high resolution crystal structures of $\mathrm{CerHb}$ in the unligated and carbomonoxy states. Although $\mathrm{CO}$ binds to the heme with an orientation different from the $\mathrm{O}_{2}$ ligand, the overall binding schemes for $\mathrm{CO}$ and $\mathrm{O}_{2}$ are essentially the same, both ligands being stabilized through a network of hydrogen bonds based on TyrB10, GlnE7, and ThrE11. No dramatic protein structural changes are needed to support binding of the ligands, which can freely reach the heme distal site through the apolar tunnel. A lack of main conformational changes between the heme-unligated and -ligated states grants stability to the folded mini-Hb and is a prerequisite for fast ligand diffusion to/from the heme. 
Keywords: nerve globin; crystal structure; heme reactivity; carbon monoxide; protein matrix tunnel

\section{Introduction}

The nerve haemoglobin $(\mathrm{Hb})$ from the nemertean worm Cerebratulus lacteus $(\mathrm{CerHb})$ is one of the smallest naturally occurring known globins, being composed of 109 amino acids instead of the $\sim 140-160$ residues typical of most globins [1,2]. Its structure shows a markedly edited globin fold with deletion of the $N$-terminal A helix, extension of the $\mathrm{GH}$ hinge region, and a shortened $C$-terminal $\mathrm{H}$ helix [3]. Both sequence and structural analyses indicate that $\mathrm{CerHb}$ is evolutionary equally distant from known globins, suggesting the presence of a specific mini-Hb family within the Hb superfamily. The affinity of $\mathrm{CerHb}$ for $\mathrm{O}_{2}$ is very close to that of mammalian myoglobins (Mbs), and its function has held to be the storage of $\mathrm{O}_{2}$ for release around axons and brain tissue during periods of burrowing under anoxic conditions [2]. From structural considerations it appears remarkable how such a small and altered globin structure can secure heme binding and the ensuing $\mathrm{O}_{2}$ storage function.

Despite the minimal size of the polypeptide chain, CerHb binds the heme group according to the stereochemical rules observed throughout the globin superfamily [3]. The heme Fe atom is regularly coordinated to the proximal His(93)F8 residue (amino acids have been identified with their topological site numbers as defined in the conventional globin fold), while on the (ligand binding) distal site $\mathrm{O}_{2}$ stabilization is achieved through a network of hydrogen bonds based on the three key residues: $\operatorname{Tyr}(11) \mathrm{B} 10, \mathrm{Gln}(44) \mathrm{E} 7$, and $\operatorname{Thr}(48) \mathrm{E} 11$. $\operatorname{Tyr}(11) \mathrm{B} 10$ and, to a lesser extent, Gln(44)E7 are the distal residues directly hydrogen-bonded to the $\mathrm{O}_{2}$ molecule, stabilizing the heme-bound form. Thr(48)E11 participates in this process by fine tuning the $\operatorname{Tyr}(11) \mathrm{B} 10 \mathrm{OH}$ group orientation relative to the $\mathrm{O}_{2}$ ligand molecule. Indeed, structural, mutagenesis, and simulation studies indicated the presence of a dynamic equilibrium between high and low affinity states of CerHb heme distal site, driven by the ability of $\operatorname{Thr}(48) \mathrm{E} 11$ to orient the $\operatorname{Tyr}(11) \mathrm{B} 10$ phenolic $\mathrm{OH}$ group relative to the ligand in such a way that the heme-bound $\mathrm{O}_{2}$ would be destabilized through electrostatic repulsion of the lone electron pairs on the $\operatorname{Tyr}(11) \mathrm{B} 10$ phenolic $\mathrm{O}$ atom [4,5]. As a result of such novel control mechanism in Hbs, based on "fractional" hydrogen bonds, the affinity of CerHb for $\mathrm{O}_{2}$ is only moderate $\left(K_{\mathrm{O} 2}=1 \mu \mathrm{M}^{-1}\right)$, and the rate of $\mathrm{O}_{2}$ dissociation is unexpectedly high $\left(k_{\mathrm{off}}=200-600 \mathrm{~s}^{-1}\right)$, contrary to the enhanced $\mathrm{O}_{2}$ affinity normally found for TyrB10-GlnE7 containing Hbs or Mbs [6].

A second distinct structural feature of $\mathrm{CerHb}$ is the presence of a long apolar tunnel that traverses the interior of the globin matrix from the heme pocket to the solvent, between the $C$-terminal ends of the $\mathrm{E}$ and $\mathrm{H}$ helices [3]. This tunnel allows a relatively unhindered access to the distal portion of the heme pocket and correlates with an unusually large association rate constant for $\mathrm{O}_{2}$ binding to $\mathrm{CerHb}$ $\left(k_{\mathrm{O} 2}^{\prime}=240 \mu \mathrm{M}^{-1} \cdot \mathrm{s}^{-1}\right)$, when compared with those of other invertebrate globins containing the Tyr-Gln active site motif ( 1 to $5 \mu \mathrm{M}^{-1} \cdot \mathrm{s}^{-1}$ ) [6]. Thus, loss of the A helix, which would block access to the channel, may have been an adaptive strategy to allow for rapid rates of $\mathrm{O}_{2}$ exchange while retaining the TyrB10-GlnE7 motif $[3,4]$. The structural and kinetic properties of the apolar tunnel have been extensively examined by X-ray crystallography, ligand binding kinetics, and molecular dynamics on 
wild type CerHb and mutants at selected sites within the tunnel (Ala(55)E18 and Leu(86)G12) [7,8]. All such data provide unambiguous experimental proof that diatomic ligands can enter and exit $\mathrm{CerHb}$ through the protein matrix tunnel, in preference to the more direct and "classic" E7 gate, typically found in $\mathrm{Mb}$ [9]. Some degree of structural heterogeneity has been highlighted at the E7 site, where both the addition of Xenon to the CerHb Leu(86)G12 $\rightarrow$ Ala mutant and oxidation of wild type $\mathrm{CerHb}$ heme iron cause the appearance of an out $\mathrm{Gln}(44) \mathrm{E} 7$ conformer, in which the amide side chain points to the solvent and appears to lower the barrier for ligand escape through the E7 gate. However, the observed kinetics suggest little entry and escape $(<25 \%)$ through the E7 pathway, presumably because the in conformer, displaying an "in" conformation for residue Gln(44)E7, is thermodynamically favoured [8].

In the work presented here, we used X-ray crystallography to determine the high resolution structures of $\mathrm{CerHb}$ in the (ferrous) unligated and in the CO-bound states (1.9 $\AA$ and $1.5 \AA$ resolution, respectively), and compared these to the oxygenated form of the protein previously reported. We show that the overall protein structure in the distal site and in the apolar tunnel is unperturbed by ligand binding; however, the $\mathrm{CO}$ and $\mathrm{O}_{2}$ ligands bind the heme Fe-atom with different orientations, while exploiting very similar H-bonding stabilization schemes within the distal site.

\section{Results and Discussion}

\subsection{Overall Structures of CerHb in the Unligated and Carbomonoxy States}

The structure of the ferrous unligated form of $\mathrm{CerHb}$ (unligated-CerHb) was refined to a general $R$-factor value of $18.3 \%$ ( $R$-free $22.1 \%$ ) for data in the $25.0-1.9 \AA$ resolution range, with ideal stereochemical parameters (root mean square deviation, r.m.s.d.) from ideal values for bond lengths $0.014 \AA$, and for bond angles $1.3^{\circ}$ ) [10]. The final model, restrained refined with isotropic $B$-factors, contains 819 protein atoms, corresponding to residues $\operatorname{Met}(0)$ to $\operatorname{Leu}(109) \mathrm{H} 20$, one heme prostetic group, 115 ordered solvent atoms (nine in two alternate positions), one glycerol molecule, two sulfate and one acetate anions (Table 1): Asp(9)B8 side-chains has been refined in a double side-chain conformation. Analysis of the electron density map at the distal iron side confirmed the absence of any ligand bound to the heme-Fe ${ }^{2+}$ atom (Figure 1a).

Restrained refinement of the carbomonoxy-form of $\mathrm{CerHb}$ (CerHb-CO) converged to a final $R$-factor value of $14.6 \%$ ( $R$-free $17.9 \%$ ) for data in the $24.7-1.5 \AA$ resolution range, with ideal stereochemical parameters (r.m.s. deviation from ideal values for bond length $0.010 \AA$ and for bond angles $1.3^{\circ}$ ) [10]. The final model contains 836 protein atoms, corresponding to residues $\operatorname{Met}(0)$ to Leu(109)H20, one heme prostetic group, 123 ordered solvent atoms, one glycerol molecule, two sulfate and one acetate anions (Table 1). Residues Met0, Asp(9)B8, Ser(76)G2, Ser(91)G17, and Leu(109)H20 were refined with double side-chain conformations. Additionally, four water molecules and one sulphate ion were modelled in two alternate positions. $B$-factors were refined anisotropically. Analysis of the electron density map at the distal iron side clearly indicates the presence of a diatomic ligand bound to the heme-Fe ${ }^{2+}$ atom (Figure 1b). 
Table 1. Data collection and refinement statistics for unligated-CerHb and $\mathrm{CerHb}-\mathrm{CO}$.

\begin{tabular}{|c|c|c|}
\hline & unligated-CerHb & CerHb-CO \\
\hline \multicolumn{3}{|l|}{ Data Collection Parameters: } \\
\hline Space group & $P 2{ }_{1} 2_{1} 2_{1}$ & $P 2{ }_{1} 2_{1}{ }_{1}$ \\
\hline \multirow[t]{3}{*}{ Cell dimensions $(\AA)$} & $a=42.1$ & $a=42.7$ \\
\hline & $b=44.3$ & $b=43.5$ \\
\hline & $c=62.1$ & $c=60.1$ \\
\hline \multirow[t]{2}{*}{ Resolution $(\AA)$} & $25.4-1.9$ & $30.5-1.5$ \\
\hline & $(2.00-1.90)$ & $(1.58-1.50)$ \\
\hline Observations & 55,282 & 129,825 \\
\hline Unique reflections & 9,482 & 18,465 \\
\hline Completeness (\%) & $98.6(100)^{a}$ & $99.6(100.0)$ \\
\hline$R$-merge ${ }^{b}(\%)$ & $20.0(35.7)$ & $8.2(31.9)$ \\
\hline$I / \sigma(I)$ & $6.0(4.2)$ & $13.4(5.7)$ \\
\hline Multiplicity & $5.8(6.4)$ & $7.0(7.3)$ \\
\hline \multicolumn{3}{|l|}{ Refinement: } \\
\hline$R$-factor ${ }^{c} / R$-free $(\%)$ & $18.3 / 22.1$ & 14.6/17.9 \\
\hline Protein atoms in the a.u. ${ }^{d}$ & 819 & 836 \\
\hline Heme prostetic group & 1 & 1 \\
\hline Carbon monoxide molecule & - & 1 \\
\hline Water molecules & 115 & 123 \\
\hline Sulfate ions & 2 & 2 \\
\hline Acetate ions & 1 & 1 \\
\hline Glycerol & 1 & 1 \\
\hline \multicolumn{3}{|l|}{ Model quality: } \\
\hline \multicolumn{3}{|l|}{ Overall $B$-factor $\left(\AA^{2}\right)$ : } \\
\hline Protein and heme & 13.9 & 13.3 \\
\hline Carbon monoxide molecule & - & 18.9 \\
\hline Ions and glycerol & 37.9 & 23.8 \\
\hline Water molecules & 25.8 & 27.1 \\
\hline \multicolumn{3}{|l|}{ Rmsd from ideal values: } \\
\hline bond lengths $(\AA)$ & 0.014 & 0.010 \\
\hline bond angles $\left({ }^{\circ}\right)$ & 1.3 & 1.3 \\
\hline \multicolumn{3}{|l|}{ Ramachandran plot $(\%)^{e}$ : } \\
\hline most favored regions & 96.8 & 96.8 \\
\hline additional allowed regions & 3.2 & 3.2 \\
\hline
\end{tabular}


Figure 1. A view of the heme cavity in (a) unligated-CerHb, and (b) $\mathrm{CerHb}-\mathrm{CO}$, including distal and proximal sites, and the $2 F o-F c$ electron density (blue mesh, contoured at $1 \sigma$ level) calculated at the end of the refinement process. Residues lining the heme pocket are shown in ball-and-stick representation (unligated-CerHb in green and CerHb-CO in yellow, respectively). The heme is seen edge on; the distal cavity is on the right of the heme.
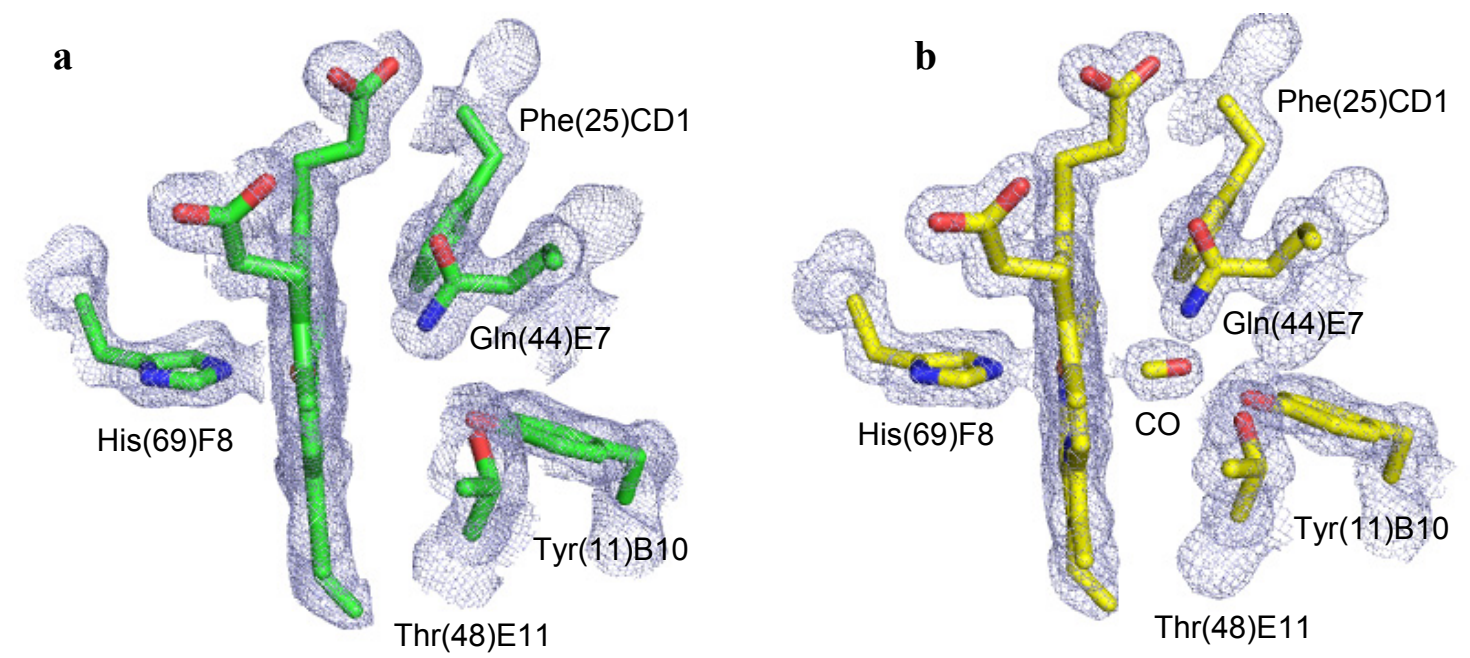

When compared to each other, the unligated-CerHb and CerHb-CO tertiary structures are very similar in their backbone, displaying a r.m.s deviation value of $0.29 \AA$, calculated over $109 \mathrm{C}_{\alpha}$ atom pairs (Figure 2). Furthermore, both structures conform well to those previously reported in the literature $[3,8]$ (see below). In particular, the CerHb-CO backbone is nearly identical to those of other ligated $\mathrm{CerHb}$ structures, such as oxygenated $\mathrm{CerHb}\left(\mathrm{CerHb}-\mathrm{O}_{2}\right)$, and aquo-met $\mathrm{CerHb}\left(\mathrm{CerHb}-\mathrm{H}_{2} \mathrm{O}\right)$, with r.m.s deviation values over $109 \mathrm{C}_{\alpha}$ atom pairs of only $0.10 \AA$ and $0.16 \AA$, respectively. On the contrary, the unligated-CerHb structure superimposed onto $\mathrm{CerHb}-\mathrm{O}_{2}$ and $\mathrm{CerHb}-\mathrm{H}_{2} \mathrm{O}$ yielded r.m.s deviation values (over $109 \mathrm{C}_{\alpha}$ atom pairs) of $0.30 \AA$ and $0.31 \AA$, respectively. Thus, despite a very good overall structural conservation, the $3 \mathrm{D}$ structures of $\mathrm{CerHb}$ in its bound states appear to cluster together based on the r.m.s.d. values.

In general, the structural deviations between $\mathrm{CerHb}$ unligated and ligated state structures can be ascribed to a contained breathing mode of the protein, whose tertiary structure shrinks slightly upon ligand binding. Indeed, if the solvent-excluded volume (i.e. the volume inside the excluded protein surface, determined using a spherical probe of $1.4 \AA$ for a water molecule) is calculated for each of the $\mathrm{CerHb}$ structures, the unligated-CerHb molecule displays a $1.5 \%-3 \%$ larger volume than $\mathrm{CerHb}$ in the ligated states. At the level of secondary structure, the only significant differences are localized at the $C$-terminus of the F helix and of the FG-hinge (residues 69-73), where the coordinate displacement of the unligated-CerHb $\mathrm{C}_{\alpha}$ backbone relative to CerHb-CO is $0.45 \AA$, and at the GH-hinge (residues 90-97), with a coordinate displacement of $0.47 \AA$. The net result is a small shift of the F helix away from the heme in the unligated structure, with the length of the Fe-His(69)F8 NE2 bond increasing from $2.06 \AA$ in CerHb-CO to $2.21 \AA$ in unligated-CerHb, while maintaining the proximal His azimuthal angle essentially unperturbed. For reference, in the CerHb- $\mathrm{O}_{2}$ and $\mathrm{CerHb}-\mathrm{H}_{2} \mathrm{O}$ structures the Fe-His(69)F8 NE2 bond length is $2.06 \AA$ and $2.05 \AA$, respectively. In the CerHb-O 2 structure the heme group shifts slightly toward the outer rim of the globin pocket $(0.29 \AA)$ relative to its position 
in unligated-CerHb, and in $\mathrm{CerHb}-\mathrm{O}_{2}$ or $\mathrm{CerHb}-\mathrm{H}_{2} \mathrm{O}$, however no significant differences are present for the iron position in the heme plane, with the iron atom only $0.08 \AA$ out-of-plane at the proximal side in the unligated-CerHb.

Figure 2. (a) Superimposition of unligated-CerHb (green) on CerHb-CO (yellow). All helical regions are labeled according to the globin fold topology, (b) Superimposition of CerHb- $\mathrm{O}_{2}$ (cyan) on CerHb-CO (yellow), and (c) $\mathrm{CerHb}^{-} \mathrm{H}_{2} \mathrm{O}$ (magenta) on CerHb-CO (yellow).

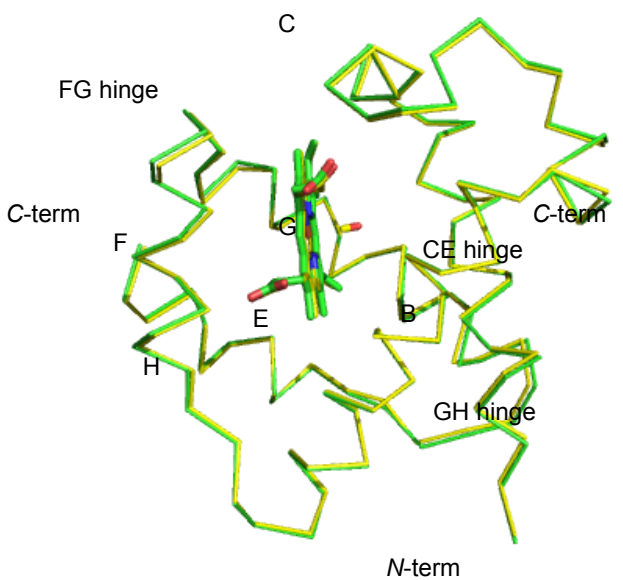

b

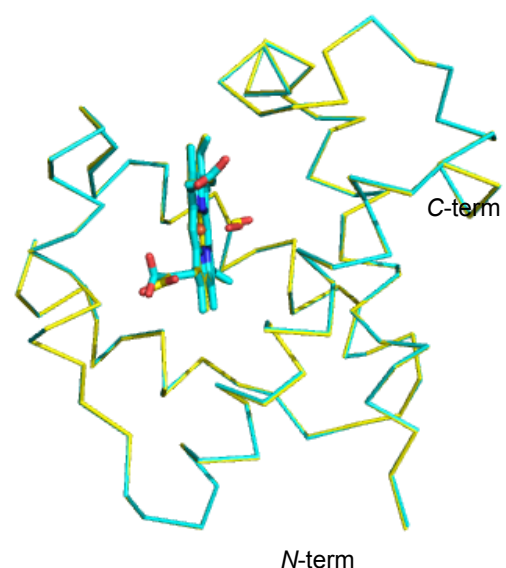

c

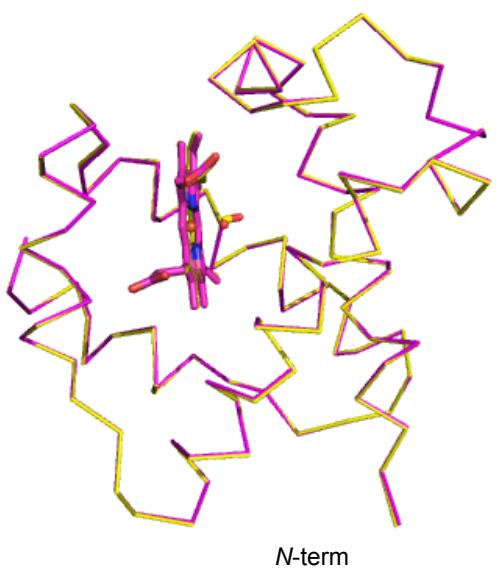

\subsection{CO Binding at the CerHb Distal Site}

The electron density clearly shows a diatomic ligand coordinated to the sixth coordination site of the heme Fe-atom in CerHb-CO (Figure 1b). On the basis of the shape of the electron density, and in view of the way crystals were prepared (see below), we identified and refined it as the exogenous $\mathrm{CO}$ molecule. The final refined model indicates an Fe-CO distance of $2.09 \AA$ and an $\mathrm{Fe}-\mathrm{C}-\mathrm{O}$ angle of $172^{\circ}$ for CerHb-CO (Table 2 and Figure 3a), comparable to that reported for Mb-CO [11,12]. Two main hydrogen bonds stabilize the heme $\mathrm{Fe}^{2+}$-bound carbon monoxide molecule. On the one hand, the carbon monoxide $\mathrm{O}$ atom is linked to the $\mathrm{Tyr}(11) \mathrm{B} 10 \mathrm{OH}$ group $(2.64 \AA)$, on the other, it is hydrogen bonded to the $\mathrm{Gln}(44) \mathrm{E} 7 \mathrm{NE} 2$ atom $(3.10 \AA)$ ). Additional polar interactions contribute to the overall structural organization of the heme distal site. Specifically, the $\operatorname{Tyr}(11) \mathrm{B} 10 \mathrm{OH}$ group is connected to the $\operatorname{Thr}(48) E 11 \mathrm{OG} 1$ atom by a strong hydrogen bond $(2.60 \AA)$, while weaker interactions connect the Tyr(11)B10 OH group to the Gln(44)E7 NE2 atom (3.06 $\AA$ ), and the Gln(44)E7 NE2 atom to the $\operatorname{Thr}(48) \mathrm{E} 11 \mathrm{OG} 1$ atom $(3.56 \AA)$. Moreover, the close contact $(3.43 \AA)$ occurring between the heme Fe-bound CO molecule (through its $\mathrm{O}$ atom) and the rim of Phe(25)CD1 should be noted, indicative of an aromatic-electrostatic contact, which further contributes to stabilization of the ligand (Figure 3a). As a result of the interlacing of side chains and hydrogen bonds, the heme Fe-bound carbon monoxide molecule is fully buried in the distal site, being totally inaccessible to solvent. If we compare the binding mode of the diatomic ligands in $\mathrm{CerHb}-\mathrm{CO}$ and $\mathrm{CerHb}-\mathrm{O}_{2}$ we notice that, despite the different binding geometry of the dioxygen molecule, the H-bonding network stabilizing the ligand is essentially the same in CerHb-CO and CerHb-O $\mathrm{O}_{2}$ (Table 2 and Figure 3b). 
Table 2. Distances between polar atoms in the distal pockets of unligated-CerHb, $\mathrm{CerHb}-\mathrm{CO}$, and $\mathrm{CerHb}-\mathrm{O}_{2}$ (covalent and hydrogen bonds are indicated as solid and dashed lines, respectively).

\begin{tabular}{lccc}
\hline & unligated-CerHb & CerHb-CO & CerHb-O \\
\hline Fe-CO $(\AA)$ & 2.09 & \\
Fe-O $(\AA)$ & & 1.94 \\
Fe-C-O angle $\left(^{\circ}\right)$ & 172 & \\
Fe-O1-O2 angle $\left(^{\circ}\right)$ & & 103 \\
Tyr(11)B10 OH---O $(\AA)$ & 2.64 & \\
Tyr(11)B10 OH---O2 $(\AA)$ & & 2.57 \\
Gln(44)E7 NE2---O $(\AA)$ & 3.10 & \\
Gln(44)E7 NE2---O1 $(\AA)$ & & 2.60 \\
Gln(44)E7 NE2---O2 $(\AA)$ & & 3.34 \\
Tyr(11)B10 OH---Thr(48)E11 OG1 $(\AA)$ & 2.76 & 2.60 & 2.59 \\
Tyr(11)B10 OH---Gln(44)E7 NE2 $(\AA)$ & 3.13 & 3.06 & 3.24 \\
Gln(44)E7 NE2---Thr(48)E11 OG1 $(\AA)$ & 3.70 & 3.56 & 3.53 \\
\hline
\end{tabular}

Figure 3. Ligand stabilization at the heme distal site of CerHb. Stereo view of the distal site region in (a) CerHb-CO (yellow) and (b) $\mathrm{CerHb}-\mathrm{O}_{2}$ (cyan). The heme iron atom is shown in orange. Hydrogen bonds are drawn as dashed lines.
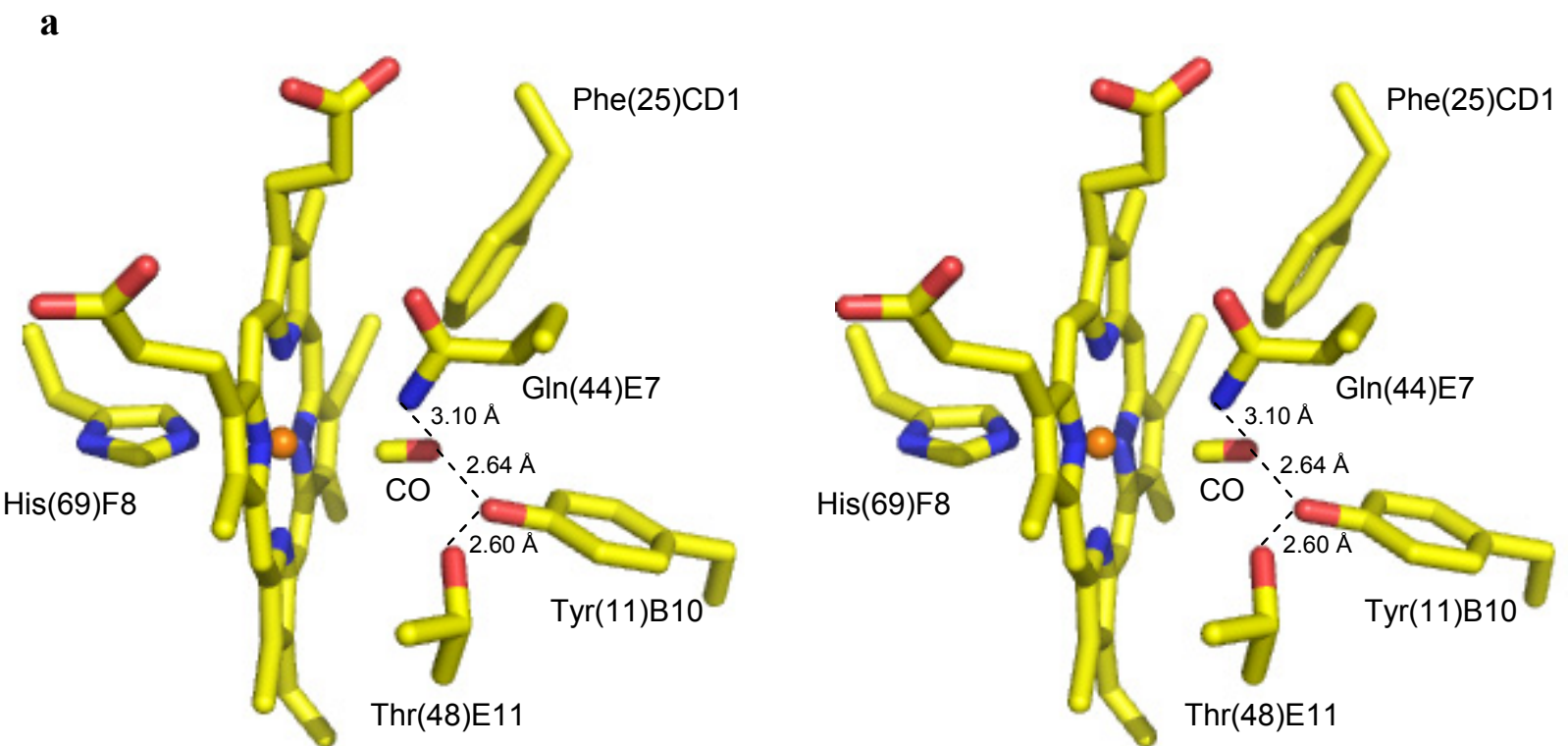
Figure 3. Cont.
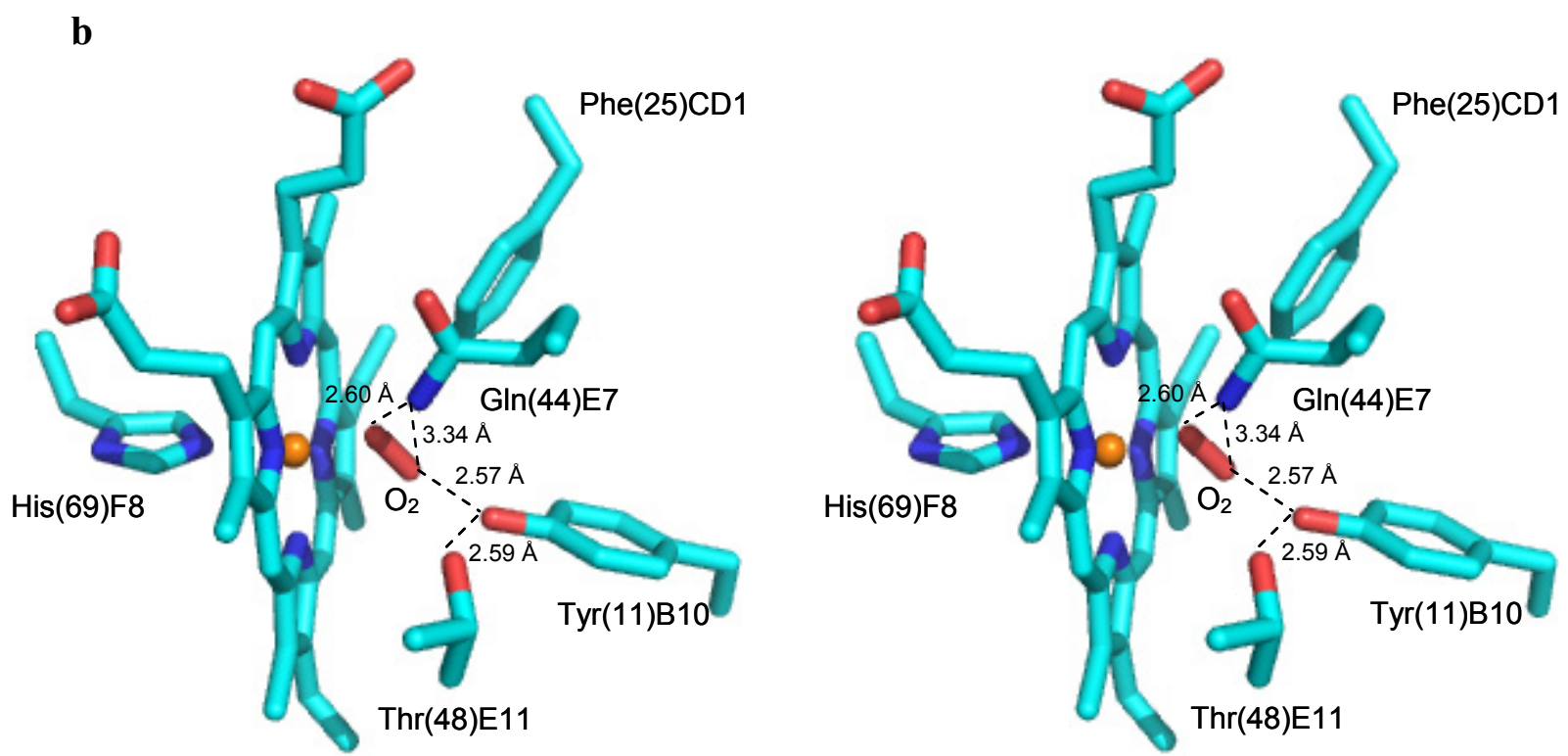

The distal site residues in the CerHb-CO and unligated-CerHb structures superimpose almost perfectly, not only in their $\mathrm{C}_{\alpha}$ positions but also in their side-chains, with only few exceptions: in the presence of the ligand the $\operatorname{Tyr}(11) \mathrm{B} 10$ side chain rotates about $4^{\circ}$ away from the centre of the distal site, while Phe(10)B9 move slightly in by a rotation of about $9^{\circ}$ around the $\mathrm{C}_{\alpha}-\mathrm{C}_{\beta}$ bond. At the same time the heme plane rotates about $4^{\circ}$ around the CHB-CHD axis, with an inwards shift of about $0.3 \AA$ (Figure 4a). Thus, we can conclude that ligand binding alters very marginally the structure of the distal site relative to the unbound state, and that the H-bonding network responsible for the stabilization of the bound ligand is substantially conserved independently of the nature of the diatomic ligand (either $\mathrm{CO}$ or $\mathrm{O}_{2}$ ).

\subsection{Ligand Diffusion Pathway through the CerHb Apolar Tunnel}

Ligand access to the heme takes place in CerHb through a wide hour-glass-shaped protein matrix tunnel connecting the distal site to a surface cleft located between the $\mathrm{E}$ and $\mathrm{H}$ helices $[3,7,8]$. The tunnel is lined with small hydrophobic residues, such as $\operatorname{Tyr}(51) \mathrm{E} 14$, Ile(52)E15, Ala(55)E18, Leu(86)G12, Leu(98), Ala(101)H12, Ile(102)H13, and $\mathrm{Ile}(105) \mathrm{H} 16$, and has a diameter that varies between 6.9 and $5.5 \AA$ in the narrowest part, which is close to residue Leu(86)G12. The tunnel end at the heme distal site is surrounded by the side chains of Val(7)B6, Phe(10)B9, and Thr(48)E11. 
Figure 4. Stereo view of the structural superimposition of CerHb-CO (yellow) and unligated-CerHb (green) (a) at the distal site, and (b) at the apolar tunnel region. Relevant residues have been shown in stick representation.

a
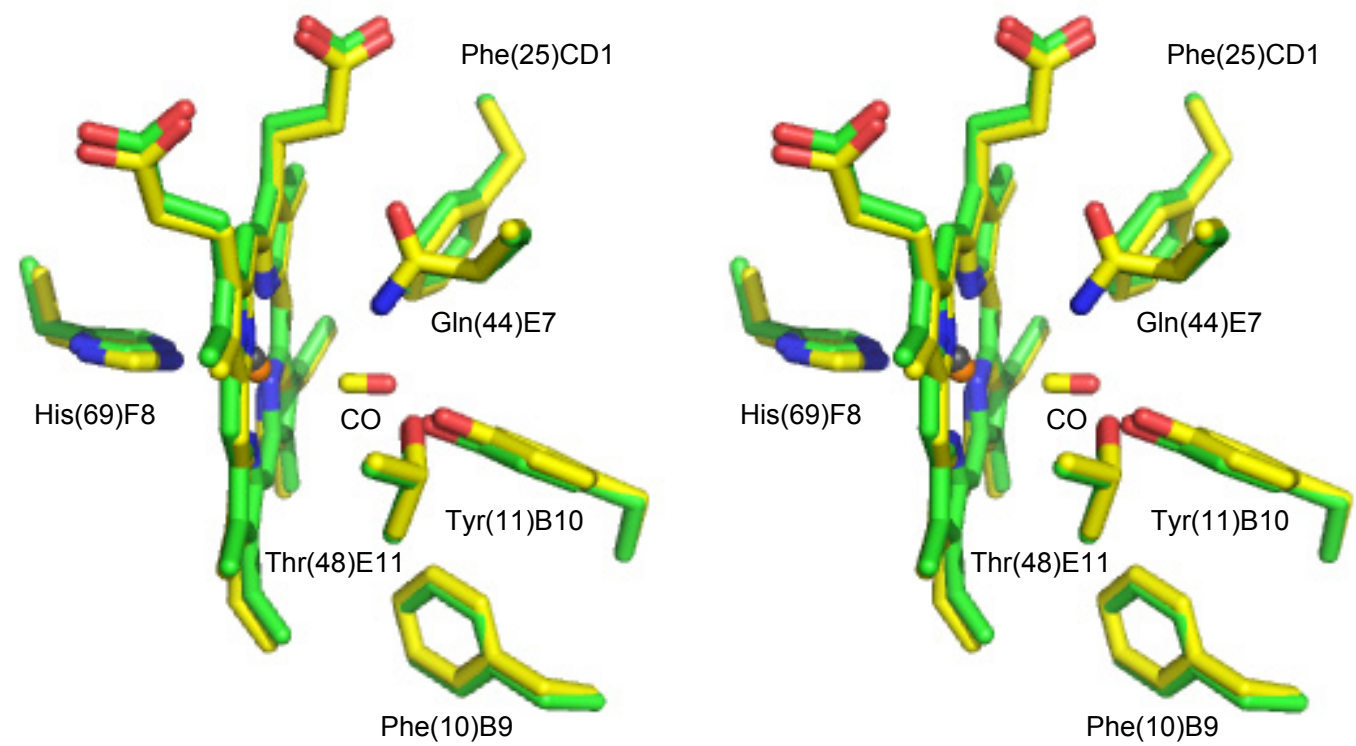

b

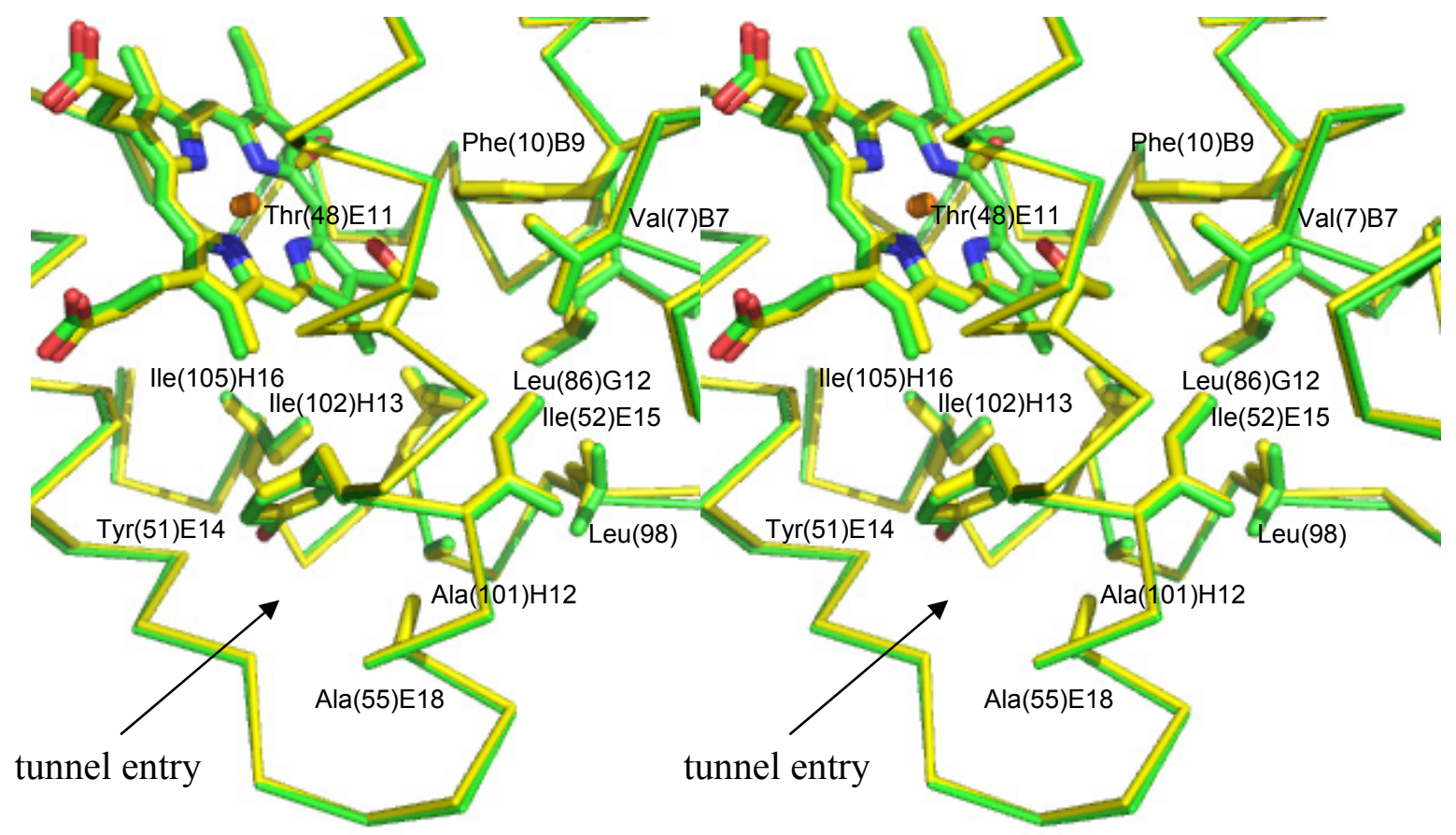

The structural comparison of unligated-CerHb and $\mathrm{CerHb}-\mathrm{CO} 3 \mathrm{D}$ structures indicates that ligand binding does not induce any relevant conformational change in the tunnel, whose lining residues match almost perfectly (Figure 4b). Comparable results, relative to the tunnel residues, are found when unligated-CerHb and $\mathrm{CerHb}-\mathrm{O}_{2}$ 3D structures are overlayed. Such findings are particularly relevant since the tunnel lining residues are all involved in determining the free energy profile for ligand entry, as calculated using dioxygen as a probe: $(i)$ free energy barrier of $\sim 5.5 \mathrm{kcal} / \mathrm{mol}$ due to steric 
restrictions posed by $\operatorname{Val}(7) B 6$, Phe(10)B9, Tyr(11)B10, and $\operatorname{Thr}(48) \mathrm{E} 11$; (ii) small ( $\leq 1 \mathrm{kcal} / \mathrm{mol})$ steric barrier near Leu(86)G12; and, (iii) a barrier of $\sim 2 \mathrm{kcal} / \mathrm{mol}$ near Ala(55)E18 at the tunnel exit to solvent. Furthermore, mutational studies showed that increasing the side chain size at Leu(86)G12 and Ala(55)E18 increases the extent of CO geminate recombination (to $\sim 50 \%$ ), and decreases both the association rate $k^{\prime} \mathrm{O} 2$ and dissociation rate $k_{\mathrm{O} 2}$ with little change in affinity [7,8]. Based on our data we can conclude that no perturbation of the apolar tunnel occurs as a result of exogenous ligand binding $\left(\mathrm{CO}\right.$ or $\left.\mathrm{O}_{2}\right)$; the tunnel therefore maintains identical structure and, likely, dynamic properties, independently of the ligation state of the protein.

\section{Experimental Section}

\subsection{Crystallization of CerHb and Derivative Preparation}

$\mathrm{CerHb}$ was expressed and purified as described previously using a synthetic gene with codon usage optimized for expression in Escherichia coli $[3,13]$. The oxygenated derivative of $\mathrm{CerHb}\left(\mathrm{CerHb}-\mathrm{O}_{2}\right)$ was crystallized by vapor diffusion techniques (protein concentration $27 \mathrm{mg} / \mathrm{mL}$ ) under conditions matching those reported in the literature [3,13]. Elongated prismatic crystals (about $0.05 \times 0.05 \times 0.3 \mathrm{~mm}^{3}$ ) grew within 1 week. The unbound form of $\mathrm{CerHb}$ (unligated-CerHb) was prepared by soaking the CerHb- $\mathrm{O}_{2}$ crystals in a stabilizing-cryo solution containing $2.8 \mathrm{M}$ ammonium sulfate, $50 \mathrm{mM}$ sodium acetate, $\mathrm{pH} 5.5,15 \%$ glycerol and $50 \mathrm{mM}$ dithionite, for $30 \mathrm{~min}$ in a sealed well. Unligated-CerHb crystals were then quickly frozen in liquid nitrogen for data collection, or transferred in a sealed well containing the same stabilizing-cryo solution but saturated with $\mathrm{CO}$ for preparation of the CO-bound derivative (CerHb-CO). After $30 \mathrm{~min}$, the CerHb-CO crystals were quickly frozen in liquid nitrogen for data collection.

\subsection{Data Collection, Phasing and Refinement}

High resolution data (1.9 $\AA$ for unligated-CerHb, and $1.5 \AA$ for CerHb-CO) were collected at $100 \mathrm{~K}$ at the European Synchrotron Radiation Facility, Grenoble, France (beam line ID23-1). These crystals belong to the orthorombic space group $P 2{ }_{1} 2_{1} 2_{1}$ (Table 1). All collected data were reduced and scaled using MOSFLM and SCALA [14,15], and phased by molecular replacement methods with the program MOLREP [16] by using the CerHb-O 2 structure as the starting model (PDB accession code 1KR7) [3]. The crystallographic refinement was performed using the program REFMAC [17], and the program COOT [18] was used for model building/inspection. The relevant data collection and refinement statistics are reported in Table 1. The program Procheck [10] was used to assess the stereochemical quality of the protein structures and the program $3 \mathrm{~V}$ to calculate the solvent-excluded volume of the protein structures [19]. Atomic coordinates and structure factors of unligated-CerHb and CerHb-CO have been deposited with PDB accession codes 4AVE, and 4AVD, respectively.

\section{Conclusions}

The present study investigated the structural changes that occur in $\mathrm{CerHb}$ upon exogenous ligand binding. The crystal structure of CerHb-CO revealed that the diatomic ligand binds to the heme $\mathrm{Fe}^{2+}$ atom with a coordination bond length of $2.09 \AA$ in a linear orientation $\left(\mathrm{Fe}-\mathrm{C}-\mathrm{O}\right.$ angle of $172^{\circ}$ ), 
markedly different if compared to the CerHb-O $\mathrm{O}_{2}$ complex, where the diatomic ligand is coordinated to the heme $\mathrm{Fe}^{2+}$ atom at $1.94 \AA$ and adopts a bent orientation, forming a $103^{\circ} \mathrm{Fe}-\mathrm{O} 1-\mathrm{O} 2$ angle in the direction of the methinic CHD atom, thus pointing to the rear end of the heme [3]. Despite the different orientations, the overall binding scheme of the bound ligands is essentially the same, and stabilized through a network of hydrogen bonds based on $\operatorname{Tyr}(11) \mathrm{B} 10, \mathrm{G} \ln (44) \mathrm{E} 7$, and $\operatorname{Thr}(48) \mathrm{E} 11$. Tyr(11)B10 and, to a lesser extent, $\mathrm{G} \ln (44) \mathrm{E} 7$ are the distal residues directly hydrogen-bonded to the ligand, stabilizing the heme-bound form. Thr(48)E11 participates in this process by fine tuning the $\operatorname{Tyr}(11) \mathrm{B} 10 \mathrm{OH}$ group orientation relative to the ligand molecule. Interestingly, comparison of the $\mathrm{CerHb}$ unligated species with $\mathrm{CerHb}-\mathrm{CO}$ and $\mathrm{CerHb}-\mathrm{O}_{2}$ reveals that binding of a ligand to the protein does not induce any dramatic conformational changes in the tertiary or in the secondary structures. As a result, both the distal site and the apolar tunnel, which provides access to the distal site from the solvent, are almost identical in structure in unligated-CerHb and in $\mathrm{CerHb}-\mathrm{CO} / \mathrm{O}_{2}$. Thus, CerHb represents an example of structural divergence within the globin superfamily where a minimal fold is able to bind stably the heme group and to take up and store $\mathrm{O}_{2}$ when the worm is in seawater, and then release it to nerve tissue when the organism burrows into mud flats and becomes anoxic [2]. To do so $\mathrm{CerHb}$ adapted its tertiary structure and heme distal site to achieve a Mb-like $\mathrm{O}_{2}$ affinity $\left(k_{\mathrm{O} 2}^{\prime}=17 \mu \mathrm{M}^{-1} \cdot \mathrm{s}^{-1}, k_{\mathrm{O} 2}=15 \mathrm{~s}^{-1}, K_{\mathrm{O} 2}=1.1 \mu \mathrm{M}^{-1}\right.$ for $\left.\mathrm{Mb}\right)$, but larger rates for $\mathrm{O}_{2}$ uptake and release $\left(k_{\mathrm{O} 2}^{\prime}=240 \mu \mathrm{M}^{-1} \cdot \mathrm{s}^{-1}, k_{\mathrm{O} 2}=180 \mathrm{~s}^{-1}, K_{\mathrm{O} 2}=1.3 \mu \mathrm{M}^{-1}\right.$ for CerHb) [4]. Moderate $\mathrm{O}_{2}$ affinity stems from the presence of a Thr residue at the E11 position (E11 is an apolar amino acid in most other animal globins) that tunes the equilibrium between high and low affinity states of CerHb heme distal site by orienting the $\operatorname{Tyr}(11) \mathrm{B} 10$ phenolic $\mathrm{OH}$ group away from the ligand [4,5]. Conversely, the need for rapid rates of $\mathrm{O}_{2}$ exchange during transient periods of hypoxia appears to have led to the creation of a large apolar tunnel between the $\mathrm{E}$ and $\mathrm{H}$ helices coupled with the concomitant loss of the A helix $[7,8]$. Thus, the Mb-like $\mathrm{O}_{2}$ affinity of $\mathrm{CerHb}$ is not merely due to a similar $\mathrm{O}_{2}$ binding mode, but to a more complex structural adaptation. In the case of $\mathrm{CO}$ binding, available data show that the main difference in the binding process can be ascribed to $\mathrm{CO}$ association to the heme, which is about 50-fold faster in $\mathrm{CerHb}$ [4]. Such a property may be related to the different ligand access paths identified in the two globins (CerHb tunnel vs. Mb E7-gate). As a result, also the $M$ ratio between the affinity constants for CO vs. $\mathrm{O}_{2}$ is about 20-fold higher in CerHb.

Our data show that no additional structural changes are needed to promote ligand binding, which can freely reach, through the tunnel, the distal site; here, the Tyr(11)B10, Gln(44)E7, and $\operatorname{Thr}(48) \mathrm{E} 11$ residues provide the H-bonding network needed for ligand stabilization.

The essentially unperturbed structure of $\mathrm{CerHb}$ in its different ligation states (but also in the absence of ligands) lends itself to few conclusive considerations. On one hand, such structural conservation may be expected for such a small protein, since conformational changes induced by ligand binding may turn into lower stability of the folded protein, or loss of heme affinity. On the other hand, a constant structure for the protein matrix tunnel is a prerequisite for efficient (fast) ligand diffusion to the heme, but also for its release. Both processes would become definitely slower if gated by conformational changes of residues limiting tunnel access/transit. Finally, and in consideration of the relatively simple architecture of $\mathrm{CerHb}$, which under any aspect is an oxygen-binding globin, a molecular tunnel devoid of functional conformational transitions can be much more easily coded during evolution. 


\section{Acknowledgments}

M.N. and M.B. are grateful to the University of Milano for supporting part of this investigation. This work was supported by a grant from Fund for Scientific research (FWO-grant $n^{\circ}$ G.0247.09) to S.D. and L.M. F.G. is supported by a fellowship from the Fund for Scientific Research (FWO).

\section{Conflict of Interest}

The authors declare no conflict of interest.

\section{References}

1. Bolognesi, M.; Bordo, D.; Rizzi, M.; Tarricone, C.; Ascenzi, P. Nonvertebrate hemoglobins: structural bases for reactivity. Prog. Biophys. Mol. Biol. 1997, 68, $29-68$.

2. Vandergon, T.L.; Riggs, C.K.; Gorr, T.A.; Colacino, J.M.; Riggs, A.F. The mini-hemoglobins in neural and body wall tissue of the nemertean worm, Cerebratulus lacteus. J. Biol. Chem. 1998, 273, 16998-17011.

3. Pesce, A.; Nardini, M.; Dewilde, S.; Geuens, E.; Yamauchi, K.; Ascenzi, P.; Riggs, A.F.; Moens, L.; Bolognesi, M. The 109 residue nerve tissue minihemoglobin from Cerebratulus lacteus highlights striking structural plasticity of the $\alpha$-helical globin fold. Structure 2002, 10, 725-735.

4. Pesce, A.; Nardini, M.; Ascenzi, P.; Geuens, E.; Dewilde, S.; Moens, L.; Bolognesi, M.; Riggs, A.F.; Hale, A.; Deng, P.; et al. ThrE11 regulates $\mathrm{O}_{2}$ affinity in Cerebratulus lacteus mini-hemoglobin. J. Biol. Chem. 2004, 279, 33662-33672.

5. Martí, M.A.; Bikiel, D.E.; Crespo, A.; Nardini, M.; Bolognesi, M.; Estrin, D.A. Two distinct heme distal site states define Cerebratulus lacteus mini-hemoglobin oxygen affinity. Proteins Struct. Funct. Bioinforma. 2006, 62, 641-648.

6. Draghi, F.; Miele, A.E.; Travaglini-Allocatelli, C.; Vallone, B.; Brunori, M.; Gibson, Q.H.; Olson, J.S. Controlling ligand binding in myoglobin by mutagenesis. J. Biol. Chem. 2002, 277, 7509-7519.

7. Salter, M.D.; Nienhaus, K.; Nienhaus, G.U.; Dewilde, S.; Moens, L.; Pesce, A.; Nardini, M.; Bolognesi, M.; Olson, J.S. The apolar channel in Cerebratulus lacteus hemoglobin is the route for $\mathrm{O}_{2}$ entry and exit. J. Biol. Chem. 2008, 283, 35689-35702.

8. Pesce, A.; Nardini, M.; Dewilde, S.; Capece, L.; Martí, M.A.; Congia, S.; Salter, M.D.; Blouin, G.C.; Estrin, D.A.; Ascenzi, P.; et al. Ligand migration in the apolar tunnel of Cerebratulus lacteus mini-hemoglobin. J. Biol. Chem. 2011, 286, 5347-5358.

9. Srajer, V.; Ren, Z.; Teng, T.Y.; Schmidt, M.; Ursby, T.; Bourgeois, D.; Pradervand, C.; Schildkamp, W.; Wulff, M.; Moffat, K. Protein conformational relaxation and ligand migration in myoglobin: a nanosecond to millisecond molecular movie from time-resolved Laue X-ray diffraction. Biochemistry 2001, 40, 13802-13815.

10. Laskowski, R.A.; MacArthur, M.W.; Moss, D.S.; Thornton, J.M. PROCHECK: a program to check the stereochemical quality of protein structures. J. Appl. Crystallogr. 1993, 26, 283-291. 
11. Kachalova, G.S.; Popov, A.N.; Bartunik, H.D. A steric mechanism for inhibition of CO binding to heme proteins. Science 1999, 284, 473-476.

12. Vojtechovsky, J.; Chu, K.; Berendzen, J.; Sweet, R.M.; Schlichting, I. Crystal structures of myoglobin-ligand complexes at near-atomic resolution. Biophys. J. 1999, 77, 2153-2174.

13. Pesce, A.; Nardini, M.; Dewilde, S.; Ascenzi, P.; Riggs, A.F.; Yamauchi, K.; Geuens, E.; Moens, L.; Bolognesi, M. Crystallization and preliminary X-ray analysis of neural haemoglobin from the nemertean worm Cerebratulus lacteus. Acta Crystallogr. D Biol. Crystallogr. 2001, 57, 1897-1899.

14. Leslie, A.G. The integration of macromolecular diffraction data. Acta Crystallogr. D Biol. Crystallogr. 2006, 62, 48-57.

15. Evans, P. Scaling and assessment of data quality. Acta Crystallogr. D Biol. Crystallogr. 2006, 62, 72-82.

16. Vagin, A.; Teplyakov, A. Molecular replacement with MOLREP. Acta Crystallogr. D Biol. Crystallogr. 1997, 66, 22-25.

17. Murshudov, G.N.; Vagin, A.A.; Dodson, E.J. Refinement of macromolecular structures by the maximum-likelihood method. Acta Crystallogr. D Biol. Crystallogr. 1997, 53, 240-255.

18. Emsley, P.; Cowtan, K. Coot: model-building tools for molecular graphics. Acta Crystallogr. D Biol. Crystallogr. 2004, 60, 2126-2132.

19. Voss, N.R.; Gerstein, M. 3V: cavity, channel and cleft volume calculator and extractor. Nucl. Acid. Res. 2010, 38, W555-W562.

(C) 2012 by the authors; licensee MDPI, Basel, Switzerland. This article is an open access article distributed under the terms and conditions of the Creative Commons Attribution license (http://creativecommons.org/licenses/by/3.0/). 